\title{
A consistent noncommutative field theory: the Wess-Zumino model
}

\author{
H.O. Girotti ${ }^{a}$, M. Gomes ${ }^{\text {b }}$, V.O. Rivelles ${ }^{\text {b,* }}$, A.J. da Silva ${ }^{b}$ \\ ${ }^{a}$ Instituto de Física, Universidade Federal do Rio Grande do Sul, Caixa Postal 15051, \\ 91501-970, Porto Alegre, RS, Brazil \\ b Instituto de Física, Universidade de São Paulo, Caixa Postal 66318, 05315-970, São Paulo, SP, Brazil
}

Received 13 June 2000; accepted 24 July 2000

\begin{abstract}
We show that the noncommutative Wess-Zumino model is renormalizable to all orders of perturbation theory. The noncommutative scalar potential by itself is non-renormalizable but the Yukawa terms demanded by supersymmetry improve the situation turning the theory into a renormalizable one. As in the commutative case, there are neither quadratic nor linear divergences. Hence, the IR/UV mixing does not give rise to quadratic infrared poles. () 2000 Elsevier Science B.V. All rights reserved.
\end{abstract}

\section{Introduction}

Noncommutative geometry has been receiving a great deal of attention in the context of string/M-theory. Initially it appeared as a possible compactification manifold of space-time [1] and led to the appearance of noncommutative quantum field theories on noncommutative tori [2-5]. More recently [6] it was shown that the dynamics of a D-brane in the presence of a $B$-field can, in certain limits, be described by a deformed gauge field theory in terms of Moyal products on space-time. Since this field theory arose from a coherent truncation of a string theory it is expected that deformed field theories are consistent by themselves. This motivated an intensive investigation of noncommutative quantum field theories on four-dimensional Euclidean and Minkowski spaces. Scalar fields

\footnotetext{
* Corresponding author.

E-mail addresses: hgirotti@if.ufrgs.br (H.O. Girotti), mgomes@ fma.if.usp.br (M. Gomes), rivelles@fma.if.usp.br (V.O. Rivelles), ajsilva@fma.if.usp.br (A.J. da Silva).
} 
[7-11], gauge fields [12-25] and supersymmetric theories [26-28] have been studied. Some two-dimensional models have also been analyzed [29-33].

A distinct characteristic of a class of noncommutative quantum field theories is the mixing of ultraviolet (UV) and infrared (IR) divergences [8] reminiscent of the UV/IR connection of string theory. For the $\phi_{4}^{4}$ massive scalar field there is an infrared quadratic singularity in the propagator at the one loop level, which jeopardizes the perturbative formulation of the theory. On the other hand, the theory has been proved to remain ultraviolet renormalizable up two loops [10] although this does not seem to hold at all orders [7]. Also, models involving complex scalar fields are not always renormalizable not even at one-loop approximation [11]. Therefore, it is relevant to understand the renormalizability properties of noncommutative field theories to find out whether they are consistent.

It has been suggested that, due to the absence of quadratic divergences in their commutative version, noncommutative supersymmetric theories may remain ultraviolet renormalizable [7,27]. The superspace formulation has already been accomplished at the classical level [27,28]. However, at the quantum level only one loop results have been reported for supersymmetric gauge theories. As in the commutative case only logarithmic divergences show up $[19,22]$.

This paper is dedicated to show that the noncommutative Wess-Zumino model in four dimensions is a consistent quantum field theory in the sense of being ultraviolet renormalizable and free of the IR/UV mixing at any arbitrary order of perturbation. This happens even though the scalar potential of the noncommutative Wess-Zumino model belongs to the class of non-renormalizable theories discussed in [11]. It is a potential typical of a F-term $\phi^{*} \star \phi^{*} \star \phi \star \phi$ (while a D-term induces $\phi^{*} \star \phi \star \phi^{*} \star \phi$ ) but nevertheless supersymmetry still eliminates all quadratic divergences. This is at the root of the renormalizability of the model.

Noncommutative field theories containing just scalar and fermion fields, as is the case in the Wess-Zumino model, are constructed from the usual Lagrangian by replacing the ordinary product by the Moyal product of fields, i.e., $A B \rightarrow A \star B$. The Moyal product is noncommutative and obeys the rule

$$
\begin{aligned}
& \int d x \phi_{1}(x) \star \phi_{2}(x) \star \cdots \star \phi_{n}(x) \\
& =\int \prod \frac{d^{4} k_{i}}{(2 \pi)^{4}}(2 \pi)^{4} \delta\left(k_{1}+k_{2}+\cdots+k_{n}\right) \\
& \quad \times \tilde{\phi}_{1}\left(k_{1}\right) \tilde{\phi}_{2}\left(k_{2}\right) \cdots \tilde{\phi}_{n}\left(k_{n}\right) \exp \left(i \sum_{i<j} k_{i} \wedge k_{j}\right),
\end{aligned}
$$

where $\tilde{\phi}_{i}$ is the Fourier transform of the field $\phi_{i}$, the index $i$ being used to distinguish different fields. In (1.1) we have introduced the notation $a \wedge b=1 / 2 a^{\mu} b^{\nu} \Theta_{\mu \nu}$, where $\Theta_{\mu \nu}$ is the anti-symmetric constant matrix characterizing the noncommutativity of the underlying space. We shall assume from now on that $\Theta_{0 i}=0$ in order to evade causality and unitarity problems [34]. 
To represent Feynman amplitudes one could either use a double line notation, as the one introduced by 't Hooft for matrix models, or single lines, which demands the symmetrization of the kernel (1.1) over the arguments of fields of the same kind. In this work we adopt the second systematics.

The paper is organized as follows. In Section 2 we present and discuss general aspects of the noncommutative Wess-Zumino model. The one loop analysis is performed in Section 3, while in Section 4 we demonstrate the renormalizability of the model to all orders of perturbation theory. Section 5 contains some final comments and the conclusions.

\section{The noncommutative Wess-Zumino model}

In four-dimensional Minkowski space-time the Wess-Zumino model is defined by the Lagrangian density $[35,36]$

$$
\begin{aligned}
\mathcal{L}= & \frac{1}{2} A\left(-\partial^{2}\right) A+\frac{1}{2} B\left(-\partial^{2}\right) B+\frac{1}{2} \bar{\psi}(i \not \partial-m) \psi+\frac{1}{2} F^{2}+\frac{1}{2} G^{2}+m F A+m G B \\
& +g\left(F A^{2}-F B^{2}+2 G A B-\bar{\psi} \psi A-i \bar{\psi} \gamma_{5} \psi B\right),
\end{aligned}
$$

where $A$ is a scalar field, $B$ is a pseudo scalar field, $\psi$ is a Majorana spinor field and $F$ and $G$ are, respectively, scalar and pseudoscalar auxiliary fields. By extending the above model to a noncommutative space one is led to the Lagrangian density

$$
\begin{aligned}
\mathcal{L}= & \frac{1}{2} A\left(-\partial^{2}\right) A+\frac{1}{2} B\left(-\partial^{2}\right) B+\frac{1}{2} \bar{\psi}(i \not \partial-m) \psi+\frac{1}{2} F^{2}+\frac{1}{2} G^{2}+m F A+m G B \\
& +g(F \star A \star A-F \star B \star B+G \star A \star B+G \star B \star A \\
& \left.-\bar{\psi} \star \psi \star A-\bar{\psi} \star i \gamma_{5} \psi \star B\right) .
\end{aligned}
$$

It should be noticed that there is only one possible extension of the cubic term $2 G A B$, to the noncommutative case, which preserves supersymmetry. It should also be emphasized that the noncommutative supersymmetry transformations are identical to the commutative ones since they are linear in the fields and no Moyal products are, therefore, involved. Hence, the extension of the theory to the noncommutative case does not alter the form of the Ward identities, which in turn implies that all fields have vanishing vacuum expectation values.

The elimination of the auxiliary fields through their corresponding equations of motion turns the bilinear terms in the Lagrangian Eq. (2.2) into the standard mass terms. On the other hand, the cubic terms produce quartic interactions which, in terms of a complex field $\phi=A+i B$, can be cast as $\phi^{*} \star \phi^{*} \star \phi \star \phi$. This potential belongs to a class of nonrenormalizable potentials, as discussed in [11]. As it will be shown below, supersymmetry saves the day turning the theory into a renormalizable one.

The Lagrangian (2.2) was also written using the superspace formalism in [27,28]. However, we will work with components fields in order to trace the effects of noncommutativity in the divergent Feynman integrals. 


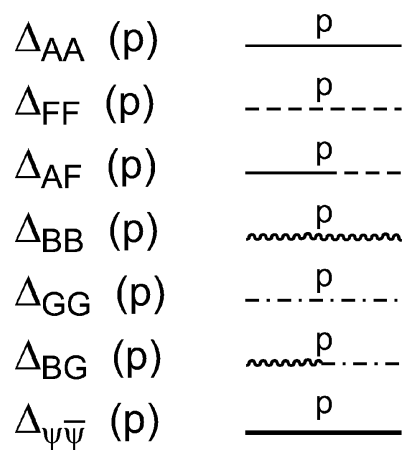

Fig. 1. Graphical representation for the propagators.

The propagators for the $A$ and $F$ fields are (see Fig. 1)

$$
\begin{aligned}
& \Delta_{A A}(p)=\Delta(p) \equiv \frac{i}{p^{2}-m^{2}+i \epsilon}, \\
& \Delta_{F F}(p)=p^{2} \Delta(p), \\
& \Delta_{A F}(p)=\Delta_{F A}(p)=-m \Delta(p),
\end{aligned}
$$

whereas the propagators involving the $B$ and $G$ fields have identical expression (i.e., they are obtained by replacing $A$ by $B$ and $F$ by $G$ ). For the $\psi$ field we have

$$
S(p)=\frac{i}{p-m} .
$$

The analytical expressions associated to the vertices are:

$$
\begin{array}{ll}
F A^{2} \text { vextex: } & i g \cos \left(p_{1} \wedge p_{2}\right), \\
F B^{2} \text { vextex: } & -i g \cos \left(p_{1} \wedge p_{2}\right), \\
G A B \text { vertex: } & 2 i g \cos \left(p_{1} \wedge p_{2}\right), \\
\bar{\psi} \psi A \text { vertex: } & -i g \cos \left(p_{1} \wedge p_{2}\right), \\
\bar{\psi} \psi B \text { vertex: } & -i g \gamma_{5} \cos \left(p_{1} \wedge p_{2}\right) .
\end{array}
$$

Due to the oscillating factors provided by the cosines some of the integrals constructed with the above rules will be finite but in general divergences will survive, the degree of superficial divergence for a generic 1PI graph $\gamma$ being

$$
d(\gamma)=4-I_{A F}-I_{B F}-N_{A}-N_{B}-2 N_{F}-2 N_{G}-\frac{3}{2} N_{\psi},
$$

where $N_{\mathcal{O}}$ denotes the number of external lines associated to the field $\mathcal{O}$ and $I_{A F}$ and $I_{B F}$ are the numbers of internal lines associated to the indicated mixed propagators. In all cases we will regularize the divergent Feynman integrals by using the supersymmetric regularization method proposed in [37]. 


\section{The one loop approximation}

It is straightforward to verify that, at the one loop level, all the tadpoles contributions add up to zero. This confirms the statement made in the previous section concerning the validity of the Ward identities.

Let us now examine the contributions to the self-energy of the $A$ field. The corresponding graphs are those shown in Fig. 2, a-e. In that figure diagrams a, b and c are quadratically divergent whereas graphs $\mathrm{d}$ and e are logarithmically divergent. We shall first prove that the quadratic divergences are canceled. In fact, we have that

$$
\begin{aligned}
\Gamma_{2 \mathrm{a}-\mathrm{c}}(A A)=-g^{2} \int & \frac{d^{4} k}{(2 \pi)^{4}} \cos ^{2}(k \wedge p)\left\{4 k^{2}+4 k^{2}-2 \operatorname{Tr}[(k k+\not p+m)(k+m)]\right\} \\
& \times \Delta(k+p) \Delta(k),
\end{aligned}
$$

where the terms in curly brackets correspond to the graphs a, b and c, respectively. After calculating the trace we obtain

$$
\Gamma_{2 \mathrm{a}-\mathrm{c}}(A A)=8 g^{2} \int \frac{d^{4} k}{(2 \pi)^{4}}\left(p \cdot k+m^{2}\right) \cos ^{2}(k \wedge p) \Delta(k) \Delta(k+p) .
$$

This last integral is, at most, linearly divergent. However, the would be linearly divergent term vanishes by symmetric integration thus leaving us with an integral which is, at most, logarithmically divergent. Adding to Eq. (3.2) the contribution of the graphs $2 \mathrm{~d}$ and $2 \mathrm{e}$ one arrives at

$$
\Gamma_{2 \mathrm{a}-\mathrm{e}}(A A)=8 g^{2} \int \frac{d^{4} k}{(2 \pi)^{4}} \cos ^{2}(p \wedge k)(p \cdot k) \Delta(k) \Delta(k+p) .
$$

To isolate the divergent contribution to $\Gamma_{2 \mathrm{a}-\mathrm{e}}(A A)$ we Taylor expand the coefficient of $\cos ^{2}(p \wedge k)$ with respect to the variable $p$ around $p=0$, namely,

$$
\begin{aligned}
& \left.8 g^{2} \int \frac{d^{4} k}{(2 \pi)^{4}} \cos ^{2}(p \wedge k) t^{(1)}(p)[(p \cdot k) \Delta(k) \Delta(k+p)]\right|_{p=0} \\
& =16 g^{2} \int \frac{d^{4} k}{(2 \pi)^{4}} \cos ^{2}(p \wedge k) \frac{(p \cdot k)^{2}}{\left(k^{2}-m^{2}\right)^{3}},
\end{aligned}
$$
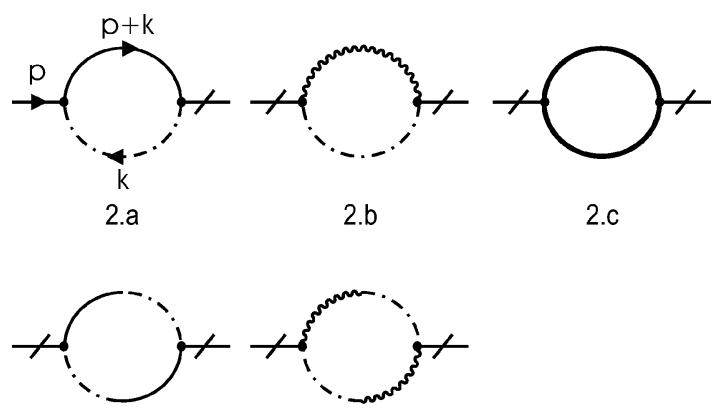

Fig. 2. One-loop contributions to the self-energy of the $A$ field. 


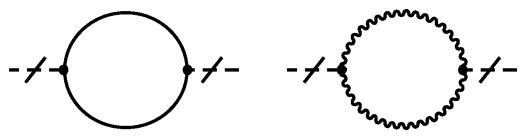

Fig. 3. One-loop corrections to the two point function of the auxiliary field $F$.

where $t^{(r)}(p)$ denotes the Taylor operator of order $r$. Since $\cos ^{2}(k \wedge p)=(1+$ $\cos (2 k \wedge p)) / 2$ the divergent part of (3.4) is found to read

$$
\Gamma_{\text {Div }}(A A)=2 g^{2} p^{2} \int \frac{d^{4} k}{(2 \pi)^{4}} \frac{1}{\left(k^{2}-m^{2}\right)^{2}} \equiv i I_{\xi} g^{2} p^{2},
$$

where the subscript $\xi$ remind us that all integrals are regularized through the procedure indicated in [37]. In the commutative Wess-Zumino model this divergence occurs with a weight twice of the above. As usual, it is eliminated by the wave function renormalization $A=Z^{1 / 2} A_{r}$, where $A_{r}$ denotes the renormalized $A$ field. Indeed, it is easily checked that with the choice $Z=1-I_{\xi} g^{2}$ the contribution (3.5) is canceled.

We turn next into analyzing the term containing $\cos (2 k \wedge p)$ in (3.4). For small values of $p$ it behaves as $p^{2} \ln \left(p^{2} / m^{2}\right)$. Thus, in contradistinction to the nonsupersymmetric $\phi_{4}^{4}$ case [8], there is no infrared pole and the function actually vanishes at $p=0$.

One may check that at one-loop the $B$ field self-energy is the same as the self-energy for the $A$ field, i.e., $\Gamma(B B)=\Gamma(A A)$. Therefore the divergent part of $\Gamma(B B)$ will be eliminated if we perform the same wave function renormalization as we did for the $A$ field, $B=Z^{1 / 2} B_{r}$. We also found that the mixed two point Green functions do not have one-loop radiative corrections, $\Gamma(A F)=\Gamma(B G)=0$.

The one-loop corrections to the two point of the auxiliary field $F$ are depicted in Fig. 3. The two graphs give identical contributions leading to the result

$$
\Gamma(F F)=-4 g^{2} \int \frac{d^{4} k}{(2 \pi)^{4}} \cos ^{2}(k \wedge p) \Delta(k) \Delta(k+p),
$$

whose divergent part is

$$
\Gamma_{\text {Div }}(F F)=2 g^{2} \int \frac{d^{4} k}{(2 \pi)^{4}} \frac{1}{\left(k^{2}-m^{2}\right)^{2}}=i I_{\xi} g^{2},
$$

involving the same divergent integral of the two point functions of the basic fields. It can be controlled by the field renormalization $F=Z^{1 / 2} F_{r}$, as in the case of $A$ and $B$. Analogous reasoning applied to the auxiliary field $G$ leads to the conclusion that $G=Z^{1 / 2} G_{r}$. However, things are different as far as the term containing $\cos (2 k \wedge p)$ is concerned. It diverges as $\ln \left(p^{2} / m^{2}\right)$ as $p$ goes to zero. Nevertheless, this is a harmless singularity in the sense that its multiple insertions in higher order diagrams do not produce the difficulties pointed out in [8].

Let us now consider the corrections to the self-energy of the spinor field $\psi$ which are shown in Fig. 4. The two contributing graphs give 


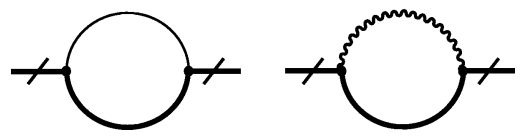

Fig. 4. One-loop contributions to the self-energy of the spinor field $\psi$.

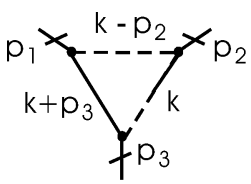

5.a

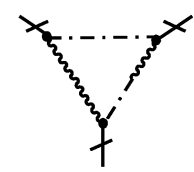

5.b

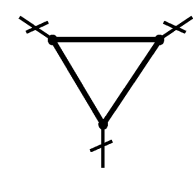

5.c

Fig. 5. Divergent graphs contributing to the three point function of the $A$ field.

$$
\begin{aligned}
\Gamma(\psi \bar{\psi}) & =4 g^{2} \int \frac{d^{4} k}{(2 \pi)^{4}} \cos ^{2}(k \wedge p) \Delta(k) \Delta(k+p)\left[(\not k+m)-\gamma_{5}(\not k+m) \gamma_{5}\right] \\
& =8 g^{2} \int \frac{d^{4} k}{(2 \pi)^{4}} \cos ^{2}(k \wedge p) \not k \Delta(k) \Delta(k+p),
\end{aligned}
$$

so that for the divergent part we get $\Gamma_{\text {Div }}(\psi \bar{\psi})=i g^{2} \not p I_{\xi}$ leading to the conclusion that the spinor field presents the same wave function renormalization of the bosonic fields, i.e., $\psi=Z^{1 / 2} \psi_{r}$. As for the term containing $\cos (2 k \wedge p)$ it behaves as $\not p \ln \left(p^{2} / m^{2}\right)$ and therefore vanishes as $p$ goes to zero.

The one-loop superficially (logarithmically) divergent graphs contributing to the three point function of the $A$ field are shown in Fig. 5. The sum of the amplitudes corresponding to the graphs $5 \mathrm{a}$ and $5 \mathrm{~b}$ is

$$
\begin{aligned}
& \Gamma_{5 \mathrm{a}+5 \mathrm{~b}}(A A A) \\
& \begin{aligned}
=96 i g^{3} m \int & \frac{d^{4} k}{(2 \pi)^{4}}\left(k-p_{2}\right)^{2} \Delta(k) \Delta\left(k+p_{3}\right) \Delta\left(k-p_{2}\right) \\
& \times \cos \left(k \wedge p_{1}+p_{3} \wedge p_{1}\right) \cos \left(p_{2} \wedge k\right) \cos \left(p_{3} \wedge k\right),
\end{aligned}
\end{aligned}
$$

while its divergent part is found to read

$$
\Gamma_{5 \mathrm{a}+5 \mathrm{~b} \operatorname{Div}}(A A A)=24 i g^{3} m \cos \left(p_{3} \wedge p_{1}\right) \int \frac{d^{4} k}{(2 \pi)^{4}}(k)^{2}(\Delta(k))^{3} .
$$

The divergent part of the graph $5 \mathrm{c}$, nonetheless, gives a similar contribution but with a minus sign so that the two divergent parts add up to zero. Thus, up to one-loop the three point function $\Gamma(A A A)$ turns out to be finite. Notice that a nonvanishing result would spoil the renormalizability of the model. The analysis of $\Gamma(A B B)$ follows along similar lines and with identical conclusions. Furthermore, it is not difficult to convince oneself that $\Gamma(F A A), \Gamma(F B B)$ and $\Gamma(G A B)$ are indeed finite.

As for $\Gamma(A \psi \bar{\psi})$ we notice that superficially divergent contributions arise from the diagrams depicted in Figs. 6a and b. In particular, diagram 6a yields 


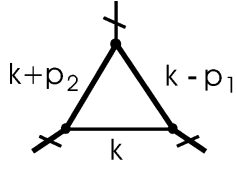

6.a

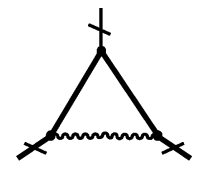

6.b

Fig. 6. One-loop contributions to the three point function $\Gamma(A \bar{\psi} \psi)$.

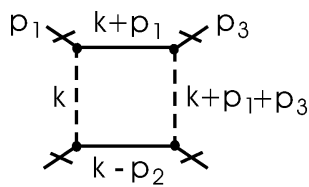

7.a

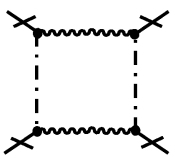

7.b

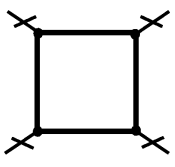

7.c

Fig. 7. Divergent graphs contributing to the four point function of the $A$ field.

$$
\begin{gathered}
\Gamma_{6 \mathrm{a}}(A \psi \bar{\psi})=8 i g^{3} \int \frac{d^{4} k}{(2 \pi)^{4}} \Delta(k) \Delta\left(p_{2}+k\right) \Delta\left(k-p_{1}\right)\left(p_{2}+\not k+m\right)\left(\not k-\not p_{1}+m\right) \\
\quad \times \cos \left(k \wedge p_{3}-p_{1} \wedge p_{3}\right) \cos \left(k \wedge p_{1}\right) \cos \left(k \wedge p_{2}\right),
\end{gathered}
$$

while $6 \mathrm{~b}$ gives

$$
\begin{aligned}
& \Gamma_{6 \mathrm{~b}}(A \psi \bar{\psi}) \\
& =-8 i g^{3} \int \frac{d^{4} k}{(2 \pi)^{4}} \Delta(k) \Delta\left(p_{2}+k\right) \Delta\left(k-p_{1}\right) \gamma_{5}\left(\not p_{2}+\not k+m\right)\left(k-\not k p_{1}+m\right) \gamma_{5} \\
& \quad \times \cos \left(k \wedge p_{3}-p_{1} \wedge p_{3}\right) \cos \left(k \wedge p_{1}\right) \cos \left(k \wedge p_{2}\right),
\end{aligned}
$$

so that the sum of the two contributions is also finite. The same applies for $\Gamma(B \psi \bar{\psi})$.

We therefore arrive at another important result, namely, that there is no vertex renormalization at the one loop level. This parallels the result of the commutative WessZumino model.

To complete the one-loop analysis we must examine the four point functions. Some of the divergent diagrams contributing to $\Gamma(A A A A)$ are depicted in Fig. 7, a-c. The analytical expression associated with the graph $7 \mathrm{a}$ is

$$
\begin{aligned}
& \Gamma_{7 \mathrm{a}}(A A A A) \\
& \qquad \begin{aligned}
=16 g^{4} \int & \frac{d^{4} k}{(2 \pi)^{4}} k^{2} \Delta(k) \Delta\left(k+p_{1}\right)\left(k+p_{1}+p_{3}\right)^{2} \Delta\left(k+p_{1}+p_{3}\right) \Delta\left(p_{2}-k\right) \\
& \times \cos \left(k \wedge p_{1}\right) \cos \left(k \wedge p_{2}\right) \cos \left[\left(k+p_{1}\right) \wedge p_{3}\right] \cos \left[\left(k-p_{2}\right) \wedge p_{4}\right] .
\end{aligned}
\end{aligned}
$$

There are five more diagrams of this type, which are obtained by permuting the external momenta $p_{2}, p_{3}$ and $p_{4}$ while keeping $p_{1}$ fixed. Since we are interested in the (logarithmic) divergence associated with this diagram, we set all the external momenta to zero in the propagators but not in the arguments of the cosines. This yields 


$$
\begin{aligned}
\Gamma_{7 \mathrm{aDiv}}(A A A A)=16 g^{4} \int \frac{d^{4} k}{(2 \pi)^{4}}\left(k^{2}\right)^{2}(\Delta(k))^{4} \cos \left(k \wedge p_{1}\right) \cos \left(k \wedge p_{2}\right) \\
\times \cos \left[\left(k+p_{1}\right) \wedge p_{3}\right] \cos \left[\left(k-p_{2}\right) \wedge p_{4}\right] .
\end{aligned}
$$

Adopting the same procedure for the other five graphs we notice that the corresponding contributions are pairwise equal. The final result is therefore

$$
\begin{aligned}
& \Gamma_{\text {Div }}(A A A A)=32 g^{4} \int \frac{d^{4} k}{(2 \pi)^{4}}\left(k^{2}\right)^{2}(\Delta(k))^{4} \cos \left(k \wedge p_{1}\right) \\
& \times\left[\cos \left(k \wedge p_{2}\right) \cos \left[\left(k+p_{1}\right) \wedge p_{3}\right] \cos \left[\left(k-p_{2}\right) \wedge p_{4}\right]\right. \\
&\left.\quad+p_{3} \leftrightarrow p_{4}+p_{2} \leftrightarrow p_{4}\right] .
\end{aligned}
$$

There is another group of six diagrams, Fig. 7b, which are obtained from the preceding ones by replacing the propagators of $A$ and $F$ fields by the propagator of the $B$ and $G$ fields, respectively. The net effect of adding these contributions is, therefore, just to double the numerical factor in the right hand side of the above formula.

Besides the two groups of graphs just mentioned, there are another six graphs with internal fermionic lines. A representative of this group has been drawn in Fig. 7c. It is straightforward to verify that because of the additional minus sign due to the fermionic loop, there is a complete cancellation with the other contributions described previously. The other four point functions may be analyzed similarly with the same result that no quartic counterterms are needed.

\section{Absence of mass and coupling constant renormalization to all orders of perturbation theory}

In the previous section we proved that up to one loop the noncommutative Wess-Zumino model is renormalizable and only requires a common wave function renormalization. Here, we shall prove that no mass and coupling constant counterterms are needed at any finite order of perturbation theory. As in the commutative case, our proof relies heavily on the Ward identities.

We start by noticing that from Eq. (1.1) it follows that

$$
\begin{aligned}
& \int d^{4} y \frac{\delta}{\delta \mathcal{O}(y)} \int d^{4} x \underbrace{\mathcal{O}(x) \star \mathcal{O}(x) \star \cdots \star \mathcal{O}(x)}_{n \text { factors }} \\
& =n \int d^{4} x \underbrace{\mathcal{O}(x) \star \mathcal{O}(x) \star \cdots \star \mathcal{O}(x)}_{n-1 \text { factors }} .
\end{aligned}
$$

In turns, this enables one to find

$$
\frac{\partial}{\partial m} Z(J)=-\frac{m}{2 g} \int \frac{\delta Z(J)}{\delta J_{F}(y)} d^{4} y-\frac{i Z(J)}{2 g} \int J_{A}(y) d^{4} y,
$$

which looks formally identical to the corresponding relation in the commutative case [37]. Here, $Z(J)$ is the Green function generating functional and $J_{\mathcal{O}}$ is the external source associated to the field $\mathcal{O}$. By collectively denoting the fields by $\phi, Z(J)$ can be cast as 


$$
Z(J)=\int D \phi \exp i\left(S+\int d^{4} x J \phi\right)
$$

where $S=\int d^{4} x \mathcal{L}$ and $\mathcal{L}$ is the regularized Lagrangian.

In terms of the 1PI generating functional $\Gamma(R)$ the identity (4.2) becomes

$$
\frac{\partial}{\partial m} \Gamma[R]=-\frac{m}{2 g} \int R_{F}(y) d^{4} y+\frac{1}{2 g} \int \frac{\delta \Gamma[R]}{\delta R_{A}(y)} d^{4} y .
$$

By taking the functional derivative with respect to $R_{F}$ and then putting all $R$ 's equal to zero we obtain

$$
m=\left.\Gamma(F A)\right|_{p^{2}=0}=\left.Z^{-1} \Gamma_{r}(F A)\right|_{p^{2}=0},
$$

where $\Gamma_{r}(A F)$ is the renormalized 1PI Green function of the indicated fields. We take as normalization conditions those specified in [37]. Specifically, $\left.\Gamma_{r}(F A)\right|_{p^{2}=0}=m_{r}$, where $m_{r}$ is taken to be the renormalized mass. Hence, $m_{r}=Z m$ implying that there is no additive mass renormalization. Through similar steps one also finds that $g_{r}=Z^{3 / 2} g$, where $g_{r}$ is the renormalized coupling constant. This implies the absence of coupling constant counterterms.

We stress the fact that, by exploiting the Ward identities, we have succeeded in generalizing to all orders of perturbation theory the one loop result concerned with the absence of counterterms different from those already present in the original Lagrangian.

\section{Conclusions}

After extending the Wess-Zumino model to the noncommutative Minkowski space, we succeeded in demonstrating, to all orders of perturbation, that the theory is free of nonintegrable infrared singularities and renormalizable. Thus, this model provides an example of a fully consistent noncommutative quantum field theory.

It shares some properties with the Wess-Zumino model. The quadratic and linear divergences are absent. Furthermore, only a wave function renormalization is needed to make the theory finite. Also, all fields exhibit the same mass as is the case in any ordinary supersymmetric theory.

On the other hand, one should notice that the commutative Wess-Zumino model can not be recovered from the noncommutative one at the limit of vanishing deformation. In fact, the limit of vanishing deformation does not exist because of logarithmic singularities.

A very important feature of the noncommutative Wess-Zumino model is that all vertices were deformed in the same way. This was essential to split the amplitudes into planar and non planar contributions in a uniform way so that the renormalizability properties of the Wess-Zumino model is always present in the planar sector. The reason for the deformed vertices to be the same is the presence of the auxiliary fields. With them all interactions are cubic. The elimination of the auxiliary fields produces cubic and quartic interactions and the vertices will be deformed in different ways. Of course, the renormalizability properties will be the same without the auxiliary fields but, surely, more difficult to prove. Supersymmetric gauge theories have cubic and quartic vertices even in the presence of 
auxiliary fields. We expect that the renormalizability proof will be much more difficult unless further simplification arise. Studies in this direction are in progress.

\section{Acknowledgements}

This work was partially supported by Fundação de Amparo à Pesquisa do Estado de São Paulo (FAPESP) and Conselho Nacional de Desenvolvimento Científico e Tecnológico (CNPq).

\section{References}

[1] A. Connes, M.R. Douglas, A. Schwarz, Noncommutative geometry and matrix theory: compactification on tori, JHEP 9802 (1998) 003, hep-th/9711162.

[2] B. Morariu, B. Zumino, Super Yang-Mills on the noncommutative torus, hep-th/9807198.

[3] C. Hofman, E. Verlinde, U-duality of Born-Infeld on the noncommutative two-torus, JHEP 9812 (1998) 010, hep-th/9810116.

[4] T. Krajewski, R. Wulkenhaar, Perturbative quantum gauge fields on the noncommutative torus, hep-th/9903187.

[5] M.M. Sheikh-Jabbari, Renormalizability of the supersymmetric Yang-Mills theories on the noncommutative torus, JHEP 9906 (1999) 015, hep-th/9903107.

[6] N. Seiberg, E. Witten, String theory and noncommutative geometry, JHEP 9909 (1999) 032, hep-th/9908142.

[7] I. Chepelev, R. Roiban, Renormalization of quantum field theories on noncommutative $R^{d}$. I: Scalars, hep-th/9911098.

[8] S. Minwalla, M. Van Raamsdonk, N. Seiberg, Noncommutative perturbative dynamics, hep-th/ 9912072.

[9] W. Fischler, E. Gorbatov, A. Kashani-Poor, S. Paban, P. Pouliot, J. Gomis, Evidence for winding states in noncommutative quantum field theory, hep-th/0002067.

[10] I.Y. Aref'eva, D.M. Belov, A.S. Koshelev, Two-loop diagrams in noncommutative $\phi_{4}^{4}$ theory, hep-th/9912075.

[11] I.Y. Aref'eva, D.M. Belov, A.S. Koshelev, A note on UV/IR for noncommutative complex scalar field, hep-th/0001215.

[12] C.P. Martin, D. Sanchez-Ruiz, The one-loop UV divergent structure of U(1) Yang-Mills theory on noncommutative $R^{4}$, Phys. Rev. Lett. 83 (1999) 476, hep-th/9903077.

[13] M. Hayakawa, Perturbative analysis on infrared and ultraviolet aspects of noncommutative QED on $R^{4}$, hep-th/9912167.

[14] H. Grosse, T. Krajewski, R. Wulkenhaar, Renormalization of noncommutative Yang-Mills theories: a simple example, hep-th/0001182.

[15] J. Madore, S. Schraml, P. Schupp, J. Wess, Gauge theory on noncommutative spaces, hep-th/ 0001203.

[16] M. Hayakawa, Perturbative analysis on infrared aspects of noncommutative QED on $R^{4}$, hep-th/ 9912094.

[17] H.B. Benaoum, Perturbative BF-Yang-Mills theory on noncommutative $R^{4}$, hep-th/9912036.

[18] M.M. Sheikh-Jabbari, Discrete symmetries (C, P, T) in noncommutative field theories, hep-th/ 0001167.

[19] A. Matusis, L. Susskind, N. Toumba, The IR/UV connection in the non-commutative gauge theories, hep-th/0002075. 
[20] J.M. Gracia-Bondia, C.P. Martin, Chiral gauge anomalies on noncommutative $R^{4}$, hep-th/ 0002171.

[21] L. Bonora, M. Schnabl, A. Tomasiello, A note on consistent anomalies in noncommutative YM theories, hep-th/0002210.

[22] I.Ya. Aref'eva, D.M. Belov, A.S. Koshelev, O.A. Rytchkov, UV/IR mixing for noncommutative complex scalar field theory II, hep-th/0003176.

[23] A. Bilal, C.-S. Chu, R. Russo, String theory and noncommutative field theories at one loop, hep-th/0003180.

[24] A. Rajaraman, M. Rozali, Noncommutative gauge theory, divergences and closed strings, hepth/0003227.

[25] A. Armoni, Perturbative dynamics of noncommutative Yang-Mills field theory, hep-th/ 0005208 .

[26] C. Chu, F. Zamora, Manifest supersymmetry in non-commutative geometry, hep-th/9912153.

[27] S. Ferrara, M.A. Lledo, Some aspects of deformations of supersymmetric field theories, hep-th/ 0002084 .

[28] S. Terashima, A note on superfields and noncommutative geometry, hep-th/0002119.

[29] H. Grosse, P. Presnajder, A treatment of the Schwinger model within noncommutative geometry, hep-th/9805085.

[30] H. Grosse, P. Presnajder, A noncommutative regularization of the Schwinger model, Lett. Math. Phys. 46 (1998) 61.

[31] E.F. Moreno, F.A. Schaposnik, The Wess-Zumino-Witten term in non-commutative twodimensional fermion models, hep-th/0002236.

[32] K. Furuta, T. Inami, Ultraviolet properties of noncommutative Wess-Zumino-Witten model, hep-th/0004024.

[33] C. Nunez, K. Olsen, R. Schiappa, From noncommutative bosonization to S-duality, hep-th/ 0005059 .

[34] J. Gomis, T. Mehen, Space-time noncommutative field theories and unitarity, hep-th/0005129.

[35] J. Wess, B. Zumino, Nucl. Phys. B 70 (1974) 39.

[36] J. Wess, B. Zumino, Phys. Lett. B 49 (1974) 52.

[37] J. Iliopoulos, B. Zumino, Broken supergauge symmetry and renormalization, Nucl. Phys. B 76 (1974) 310. 